\title{
Tritium clouds environmental impact in air into the Western Mediterranean Basin evaluation
}

\author{
P. Castro M. Velarde J. Ardao J.M. Perlado L. Sedano
}

\begin{abstract}
A B S T R A C T
The paper considers short-term releases of tritium (mainly but not only tritium hydride (HT)) to the atmosphere from a potential ITER-like fusion reactor located in the Mediterranean Basin and explores if the short range legal exposure limits are exceeded (both locally and downwind). For this, a coupled Lagrangian ECMWF/FLEXPART model has been used to follow real time releases of tritium. This tool was analyzed for nominal tritium operational conditions under selected incidental conditions to determine resultant local and Western Mediterranean effects, together with hourly observations of wind, to provide a short-range approximation of tritium cloud behavior. Since our results cannot be compared with radiological station measurements of tritium in air, we use the NORMTRI Gaussian model. We demonstrate an overestimation of the sequence of tritium concentrations in the atmosphere, close to the reactor, estimated with this model when compared with ECMWF/FLEXPART results. A Gaussian "mesoscale" qualification tool has been used to validate the ECMWF/FLEXPART for winter 2010/spring 2011 with a database of the HT plumes. It is considered that NORMTRI allows evaluation of tritium-in-air-plume patterns and its contribution to doses.
\end{abstract}

\section{Introduction}

Present devices as ITER manage amounts of tritium in the order of kilograms. Tritium release guidelines at ITER in normal conditions; less than $1 \mathrm{~g}$ of tritium hydride (HT) per year, less than $0.1 \mathrm{~g}$ per year of tritium oxide (HTO), are known to be below but within the order of magnitude of Committed Effective Dose Equivalent (CEDE) dose prescribed exposure limits by international regulations ( $1 \mathrm{mSv}$ per year) [ICRP-60]. However a future potential decrease of those prescribed limits could mean potential dramatic impact on fusion plants design requirements and correspondent costs. Present dose assessments deterministic and probabilistic schemes can be assumed at present. Such models are mainly based on the assumption of Gaussian models for discharges and the consideration of diverse atmosphere stability classes using the results in similar scenarios using NORMTRI code [1].

In previous papers real time assessments on tritium plumes and its patterns were presented $[1,2,9,10]$. This paper presents the evaluation of the radiological environmental impact of tritium routine emission chronic results (after emission to the atmosphere, adsorption and reemission from soil) for same source term ( $1 \mathrm{~g} /$ year of HT or $1 \mathrm{~g} /$ year of HTO) in the surroundings of the Plant, mainly but not only in the exclusion area. The doses outputs associated to the secondary phase are the object of this study [11].

Therefore, present dose assessments should be assumed as overestimated by principle. Today, highly sophisticated atmospheric models are open to the use of real-time numerical meteorologi$\mathrm{cal} /$ geographical synoptic data; e.g.: 91 atmospheric vertical levels (of pressure) and $25 \mathrm{~km}$ terrestrial meshing. The use of real-time data and precise boundary conditions for both, atmosphere and soil, together with Lagrangian dispersion models and its coupling with sophisticated tritium dose transfer schemes at secondary phases by ingestion and/or inhalation, can determine the two chemical tritium forms (HT or tritium hydride and HTO or tritium oxide) environmental impact at any low level of required limit. There are coupling procedures of wind, solar radiation, precipitation, relative humidity; temperature fields' records of input European Centre for Medium range Weather Forecast (ECMWF) [4,5] with the Lagrangian dispersion model FLEXPART. ECMWF is twofold; a real-time processing meteorological data source (or rich data assimilation system) and a quasi-Lagrangian forecasting model.

As discussed, the final qualification of the refinement soundness of the proposed method shall be established by the comparison of the predictions between Gaussian NORMTRI and ECMWF/FLEXPART Lagrangian modeling approaches and with their 
Table 1

Evolution of ECMWF model resolution before TL1279 under development [3].

\begin{tabular}{llll}
\hline Year & Name & Spatial resol. & Vertical levels \\
\hline 1985 & TL106L19 & 106 & 19 \\
1991 & TL213L31 & 213 & 31 \\
1998 & TL319L50 & 319 & 50 \\
2000 & TL511L60 & 511 & 60 \\
2006 & TL799L91 & 799 & 91 \\
\hline
\end{tabular}

confrontation with available experimental records. Roadmap of the ECMWF/FLEXPART development needs to qualify the new proposed schemes for dose assessments.

\section{ECMWF numerical model and FLEXPART Lagrangian dispersion model}

\subsection{ECMWF}

The ECMWF Integrated Forecast System (IFS) consists of a general circulation model, a data assimilation system and an ensemble forecast system. ECMWF, is a Medium range Weather Forecast Model (with forecast fields valid until $240 \mathrm{~h} / 10$ days). The numerical scheme will consist in a spectrum (with triangular truncation, 799 waves around a great circle on the globe; 91 vertical levels between the earth's surface and $1 \mathrm{~Pa}$, about $85 \mathrm{~km}$ ). It has a semi-Lagrangian formulation. The model has 76 million grid points separated by about $25 \mathrm{~km}$, in the horizontal around the globe. In this work actual IFS consist we use $50 \mathrm{~km}$ resolution.

Table 1 shows the evolution of ECMWF model resolution since 1985. In particular, the new cycle of IFS model that has higher resolution upgrade (cycle labeled as $36 \mathrm{r} 1$ ) a deterministic forecast and analysis horizontal resolution increased from T799 to T1279 has not been used here [3]. The variables at each grid point are: wind (including vertical velocity), temperature, humidity, and cloud water and ice and cloud fraction, ozone (also pressure at surface grid-points). Data assimilation is hourly in surface observations and 3 hourly for SYNOP observations. Aerological soundings (at certain vertical levels) made in specific points at 00 and 12 UTC. Finally there is a variational assimilation of Radiative Transfer Models for satellite data.

\subsection{Adiabatic formulation of ECMWF}

The adiabatic formulation of the ECMWF model shows six equations. They are the Euler equations for a moist atmosphere in a rotating sphere. Momentum equations in spherical coordinates $(\lambda$, $\theta, r)$; continuity equation, thermodynamic equation and Euler equations in shallow atmosphere (Hydrostatic Primitive Equations or HPE) approximation are far from the scope of this paper. In detail they are in Fig. 1.

To summarize we have 2 diagnostic equations (gas law and hydrostatic equation) and the following 4 forecast equations:

(a) Continuity equation (mass conservation required to determine vertical wind speed and changes in surface pressure).

(b) The equation of motion or 3D momentum equation (describes the acceleration deceleration of the speed of an air parcel due to pressure gradient force, and how Coriolis force contributes to affect the direction. Effects of turbulent drag, gravity wave breaking and momentum transport due to moist convection are already included).

(c) The thermodynamic equation (expresses how an air parcel's temperature is changing by adiabatic cooling or warming during vertical displacements. Also included physical processes:

\begin{tabular}{|c|c|}
\hline$\frac{D \vec{V}}{D t}=-2 \vec{\Omega} \times \vec{V}-\frac{1}{\rho} \nabla p+\vec{g}+\vec{F}_{r}$ & 3D Momentum equation \\
\hline$\frac{\partial \rho}{\partial t}+\nabla \cdot(\rho \vec{V})=0 \Leftrightarrow \frac{D \rho}{D t}+\rho \nabla \cdot \vec{V}=0$ & Continuity equation \\
\hline$c_{v} \frac{D T}{D t}+L \frac{D q}{D t}+p \frac{D \alpha}{D t}=Q$ & Thermodynamic equation \\
\hline$\frac{D q}{D t}=P_{q}$ & Humidity equation \\
\hline$\frac{D X_{i}}{D t}=P_{s i}$ & $\begin{array}{c}\text { Transport equation of various } \\
\text { physical/chemical species }\end{array}$ \\
\hline$p=\rho R T$ & Equation State \\
\hline
\end{tabular}

Fig. 1. ECMWF adiabatic formulation [4].

condensation, evaporation, turbulent transport and radiative effects).

(d) The conservation of moisture (content except for losses due to condensation or precipitation, or gains by evaporation from clouds and rain, or from oceans and continents).

Attention should be paid on the fact that the pressure is the vertical coordinate in the hydrostatic system; as it is used within the HPE. Also it can be worthwhile to note boundary conditions. Governing equations has to be solved in the lower boundary of the atmosphere (surface of the earth) which is a material boundary (air parcel cannot cross it!) and velocity component perpendicular to surface has to vanish $(w=0)$. Unfortunately the topography of the earth is far from flat (there are many mountain systems inland the Iberian Peninsula) making quite hard to apply the lower boundary condition. The solution is to use a terrain-following vertical coordinate so called hybrid sigma-pressure (vertical) coordinate, where the coordinate surfaces are sigma surfaces near the ground, gradually become more horizontal with increasing distance from the surface and turn into pure pressure surfaces into the stratosphere [3].

ECMWF requires a spectral transform method [5] with Fast Fourier Transform and Legendre transform between full and reduced grids. The spectral representation has advantages as those related with analytical computation of horizontal derivatives (they produce pressure gradient terms very accurate and there is no need to stagger variables on the grid) and the spherical harmonics are eigenvectors of the Laplace operator and applying high-order diffusion is very easy. The disadvantage is the computational cost of the Legendre transform. It is high and grows faster with increasing horizontal resolution than the cost of the rest of the model [5]. Therefore we have selected for the mentioned coupling the use of T511L60 with a reliable cost profile, mainly but not only, in the transpositions.

\subsection{ECMWF/FLEXPART coupling}

We use the model for detecting Mediterranean cyclogenesis responsible for organizing the inflow of wet and warm air over the Iberian Peninsula. We consider this type of meteorological situation as responsible for potential dispersion or diffusion of the plumes at largest scales. At present stage of tool developments, and among many other fields, we use wind forecast fields from IFS as linked input in the Lagrangian system, which reading run specifications integrate a cluster of particle positions. 
The IFS output couples with FLEXPART as input.

FLEXPART is a Lagrangian particle dispersion one and simulates in short range and the mesoscale transport, diffusion, dry and wet deposition, and radioactive decay of tracers. Dispersion from the release point has been obtained by fixing the level in $10 \mathrm{~m}$. Tritium has been assumed in present work. The model can calculate backward dispersion of a parcel in different heights of the atmosphere as a classic product. In this work we produce new products using coupled FLEXPART forward in time, to simulate the dispersion of tracers from the release point. For this purpose ECMWFDATA software has been used modified for Vandellós as point of release of a possible fusion reactor site. This software requires supplying the ATM model FLEXPART with input data describing the state of the atmosphere and thus the current transport conditions.

In Fig. 2 we can see the limited area selected and the fusion reactor site marked with a cross. We are interested in the short range dispersion, which implies air HT forecasting with implies air HT forecasting with ECMWF/FLEXPART $24 \mathrm{~h}$ ahead). The representation of plume trajectories can be associated to the coordinates of maximum concentrations.

The example of diffusion of HT on 26-Jan-2011 at the levels of 10, 30 and $60 \mathrm{~m}$ by the Mediterranean Sea (Fig. 3 ) and their trajectories of the quasi-similar HT plumes when diffusion from the release point-source at V-Plant may be due to lack of orographic effects (Von Karmann vortices and so on). This allows us to select the best optimization of the diffusion in the atmosphere with "very similar" trajectories in the first $24 \mathrm{~h}$. Other scenarios much more complex should be ad hoc for those HT plumes coming from Vandellós with diffusion by inland Iberian Peninsula.

\section{Comparison between ECMWF/FLEXPART model and NORMTRI model}

On one hand FLEXPART is a Lagrangian model of atmospheric contaminants, here adapted in both chemical forms of atmospheric tritium, using as input data the outputs of the numerical weather prediction model of the ECMWF. As this model has 60 levels in the vertical of the atmosphere (an horizontal resolution of $50 \mathrm{~km}$ in the horizontal) the coupled ECMWF/FLEXPART became a proposed tool to better describe the variability of weather behavior (taking into account in instance the wind, not only near the ground, but also in all vertical levels of the atmosphere, from the boundary layer to the troposphere and the stratosphere).

We made a HT/HTO data base [8] by using the short range forecast from 3 to $24 \mathrm{~h}$, with a frequency of $3 \mathrm{~h}$.

The area of interest should be synoptic or mesoscalar [4]. In our case ECMWF/FLEXPART we have selected the one ranging from latitude $38^{\circ}$ North, longitude $1^{\circ} 27^{\prime}$ West, to the latitude $43^{\circ}$ North longitude $7^{\circ} 38^{\circ}$ East; allowing us for the evaluation of tritium plumes into this particular area over the Mediterranean Sea. It is important to understand FLEXPART uses as input all surface and height observations of temperature, humidity, precipitation and wind speed and wind direction that are required in the analysis for first guess of the model fields of those variables (allowing for forecasting in real time). It already gives some products concerning depositions in soil (dry and wet).

NORMTRI [12] has been developed to calculate the behavior of tritium in the environment released into the atmosphere under normal operation of nuclear facilities. NORMTRI is based on the statistical Gaussian dispersion model ISOLA, which calculate the activity concentration in air near on the surface contamination due to dry and wet deposition at specified locations in a polar grid system. ISOLA requires a different parametric meteorological statistics derived from one year local synoptic recordings (at Vandellós, with determined frequency: of $1 \mathrm{~h}$ averages of wind speed, wind direction, stability class and precipitation intensity. The great value of
NORMTRI opens the possibility to choose several dose calculations procedures ranging from the equations of the European regulatory guidelines based in an equilibrium approach. NORMTRI is applied to radial zones centered in Vandellós. NORMTRI uses 3 climatological sequences. First sequence: winter period from the first of January to $31 \mathrm{March}$; second period called vegetable (growing) period, ranges from April 1 to October the 15th; third period: start on October 16 ending by December 31st [5-7]. The reemission periods (after plume passage over soil and "makes a reflexion") in NORMTRI for HT are $144 \mathrm{~h}$; for HTO is $70 \mathrm{~h}$. Soil has 4 layers, but reemission in the soil layer is produced from 0 to $5 \mathrm{~cm}$ of depth. The area for verification can be different of the grid of this Gaussian model. We decide to select a radial special area centered in Vandellós Fusion Plant and execution of models is reviewed by specific zones for validation. NORMTRI has been used for dose predictions.

To summarize this model and ECMWF/FLEXTRA has been compared with NORMTRI [1] and as a conclusion it was detected a NORMTRI apparent overestimation of ECMWF_FLEXTRA HT values close reactor (when HT values are considered for all year NORMTRI values are under ECMWF/FLEXPART values). For all distances ECMWF_FLEXTRA give real time values. The value of HT is calculated in real time for defined patterns [2]. The real time values escalated to the year are shown in Fig. 4. In this case there is a NORMTRI sub estimation of HT concentration values at downwind distances.

\section{EDES under PENTA level 3 requirements for precautionary measures taken from NORMTRI. ECMWF/FLEXPART required model}

Since our results cannot be compared with radiological station measurements of tritium in air, we use the NORMTRI Gaussian model. It is considered that NORMTRI allows evaluation of tritiumin-air-plume patterns and its contribution to doses. E.g. in order to validate the ECMWF/FLEXPART model it has been determined, in routine conditions of ITER the Most Exposed Individual (MEI) remains under defined values in the PENTA protocol (or Emergency Plan for Tarragona) [5] as precautionary measurements.

Some examples of Equivalent Effective Dose - EDE (Sv) for defined zones 0 , I and II $[1,2]$ in the closest $30 \mathrm{~km}$ to the reactor were presented. When we made the validation of the model ECMWF/FLEXPART with a new data base of tritium concentrations for November 2010 to March 2011 we had used special air patterns for tritium transport from source to the atmosphere low levels in the boundary layer in form of "tritium clouds" if elemental HT or tritium oxide [3]. We use defined tritium cloud patterns [2]. Concerning source term we assume major concentration activity of the tritium species should be in the very low levels. Therefore we have selected $10 \mathrm{~m}$ for obtaining ECMWF/FLEXPART products of the emission release of tritium (when in continuous displacement in the boundary layer). The diffusion of atmospheric tritium can be very different, as a function of the water vapor tritium clouds development and movement (stratified or convective, horizontal or with vertical ascendant or descendent air currents).

\section{Contribution to dose in all of the pathways}

By following test cases (100\% HT and 100\% HTO) for ITER-V at $30 \mathrm{~m}$, we represented contribution of exposure pathways to EDE. Taking into account PENTA urgent actions (those to be adopted in a fast way in order to be efficient, otherwise their efficiency - concerning population health - would be significantly decreased) that would be applied during a short period of time. Within those urgent precautionary measures there are mainly three; confinement, radiological prophylaxis and evacuation. 

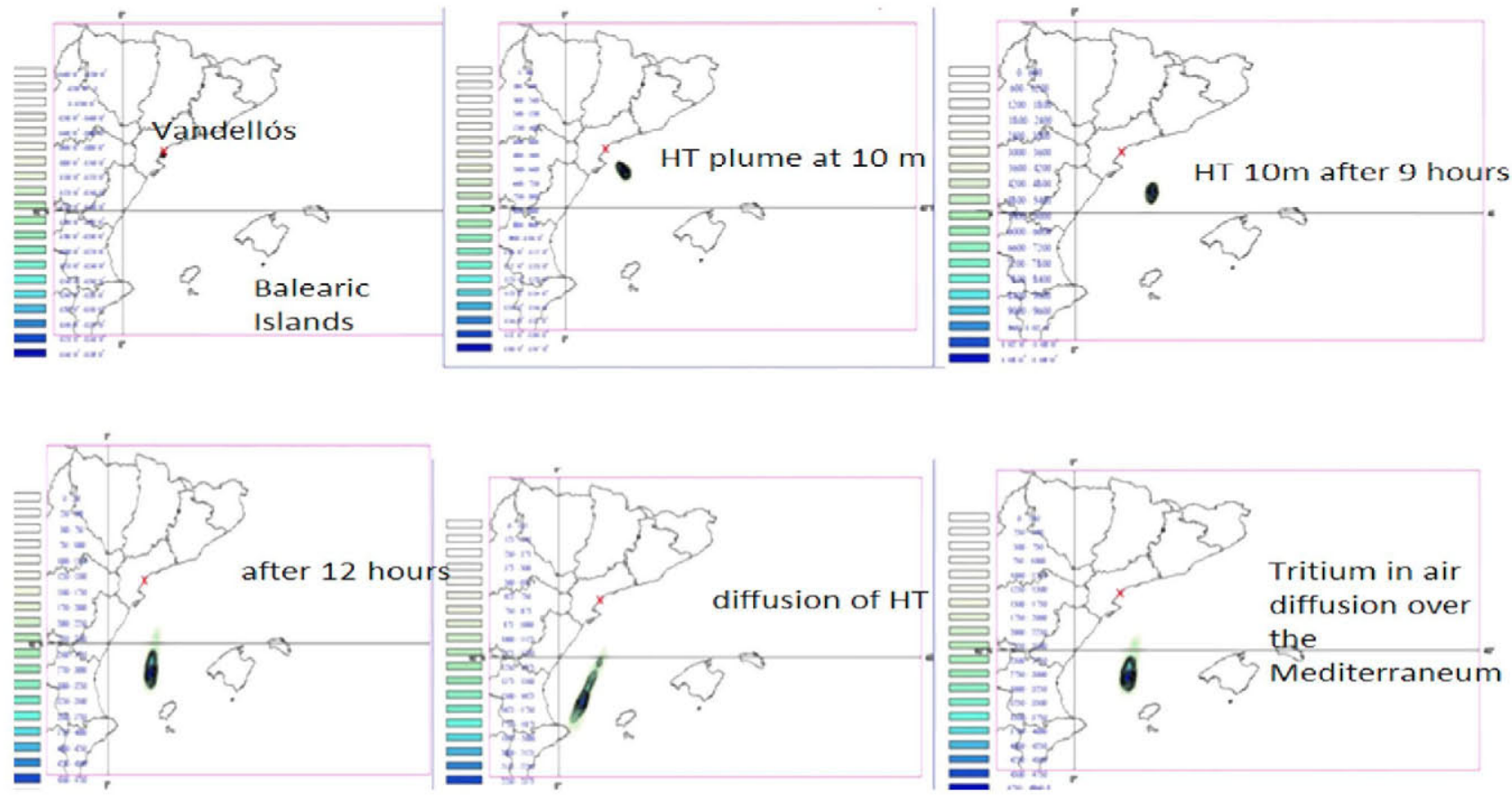

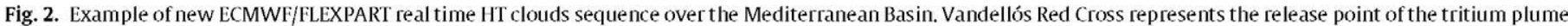
at $10 \mathrm{~m}$.

Effective Dose (EDE) from NOMTRI study case was studied by Castro et al. [1]. NORMTRI executed for ITER reactor tritium effluents HT/HTO at 30 level in routine, has given Effective Dose (EDE) by contribution of exposure pathways (percentage of inhalation, percentage of ingestion; and percentage of inhalation due to the reemission. The question was if fusion routine delivery of tritium in atmosphere produces effective doses over $10 \mathrm{mSv}$ (and therefore confinement should be taken into account)? Similarly can fusion routine delivery of tritium in atmosphere produce effective doses over $100 \mathrm{mSv}$ and therefore radiation prophylaxis (tiroides) should be taken into account? NORMTRI study case results with contributions of exposure pathways to EDE have been obtained and they were represented for both chemical tritium forms [1]. We found all values less than $10 \mathrm{mSv}$ (and under $1 \mathrm{mSv} / \mathrm{year}$, the ITER exposure to radiation limit in the French regulation for public) in the PENTA zones. Therefore the answers to our questions were negative, in other words at all radial distances the precautionary PENTA measurements, both for confinement, both for prophylaxis, in those emergency planning zones (EPZ) are not required. This is due correspondent doses are under the regulatory limits.

Finally for evaluation of the tritium-clouds environmental impact in air into the Western Mediterranean Basin we have to come back NORMTRI, due NORMTRI uses required climatologically sequences (one agricultural year). Therefore we can consider all of the pathways to make contribution at doses in both chemical form of tritium: HT and HTO, doses in vegetation, meat and milk in the normal diet of Mediterranean Basin people. The inhalation doses

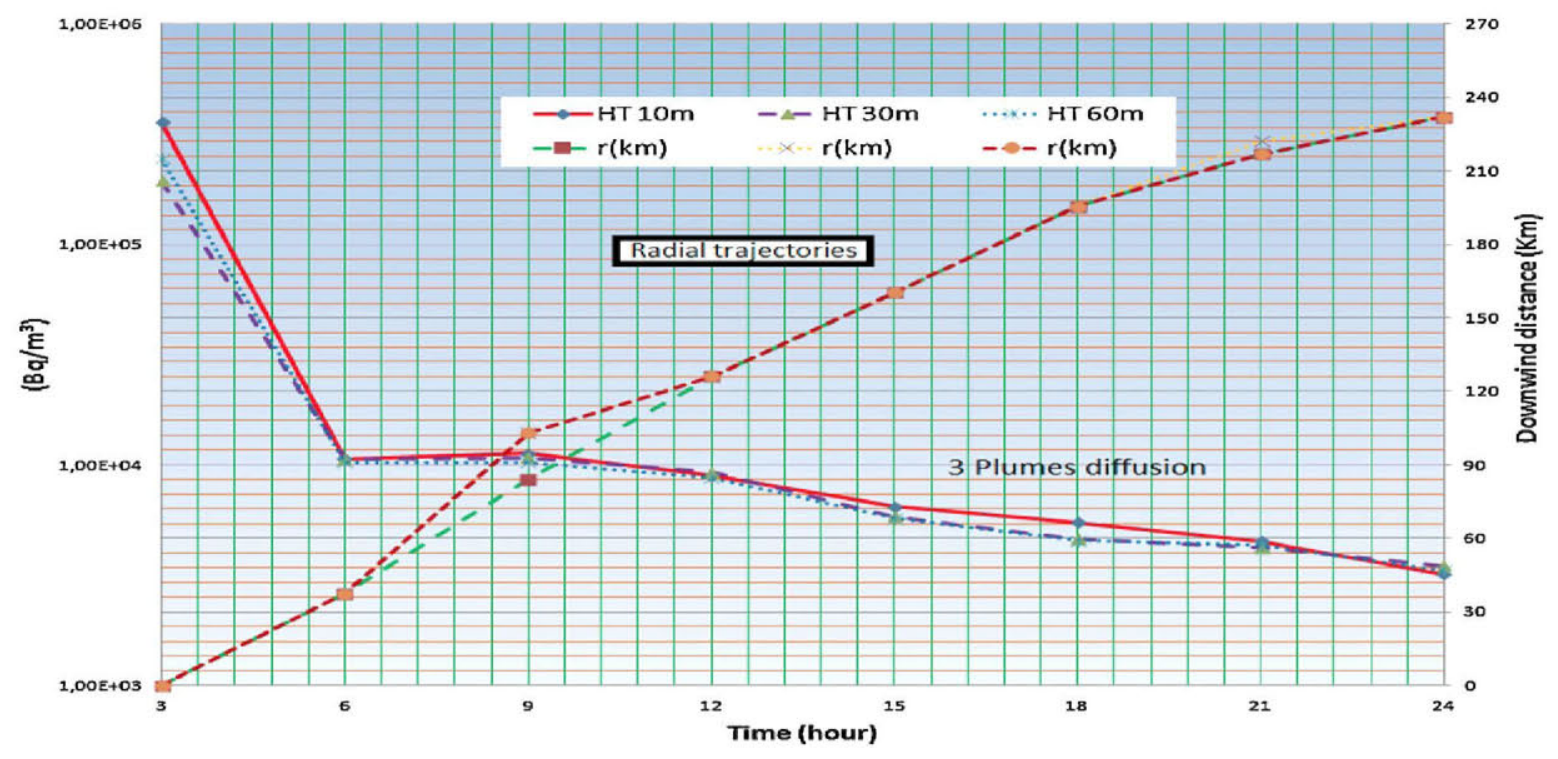

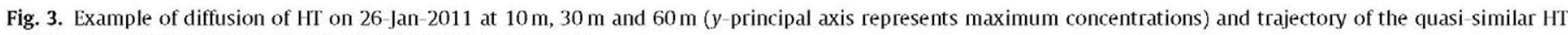
plumes when diffusion from the release point-source at V-Plant. 

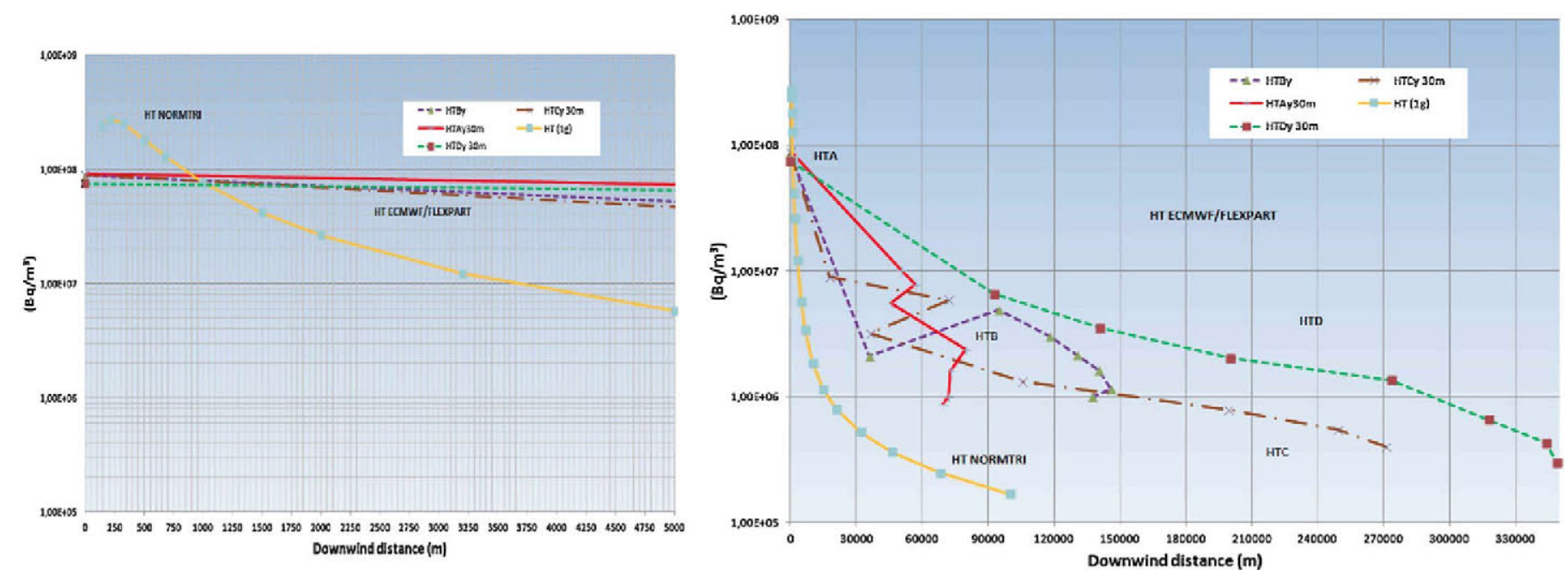

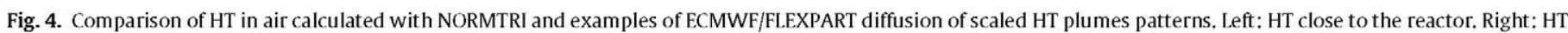
at downwind distances.

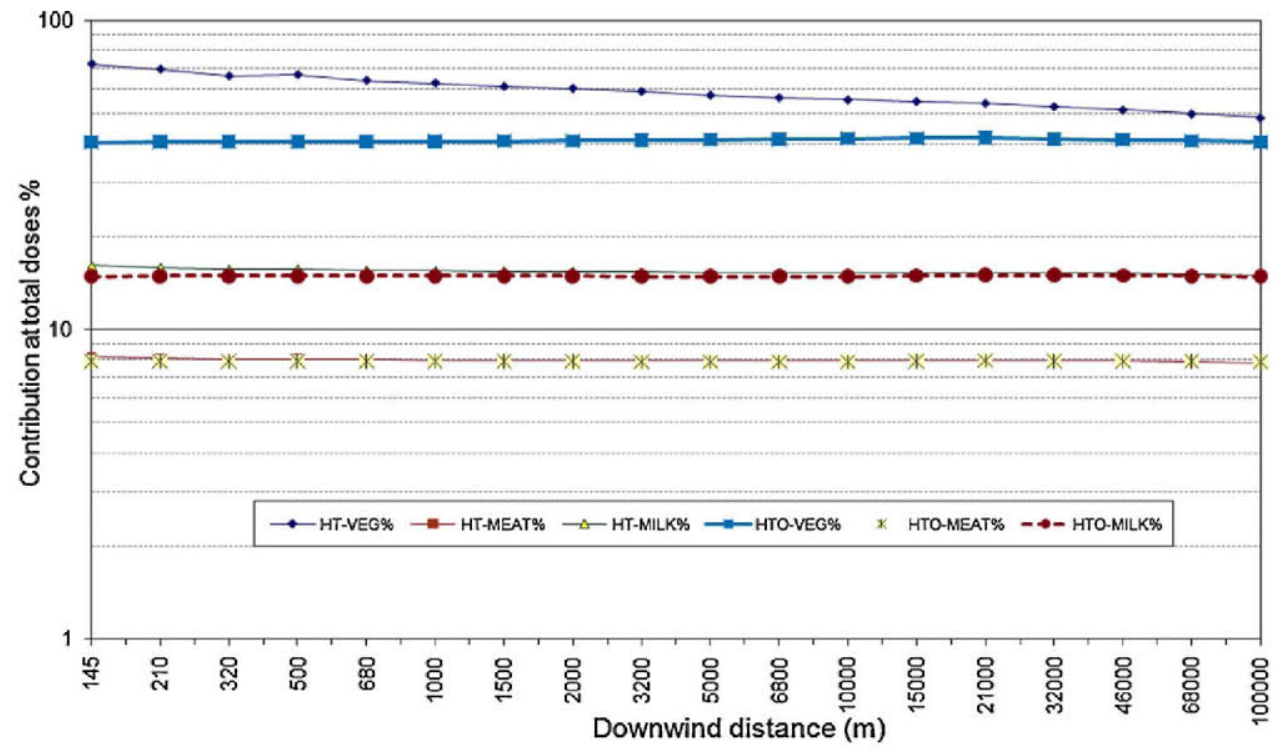

Fig. 5. Contribution at total doses coming from $\mathrm{HT}$ in vegetation, meat and milk by downwind distance.

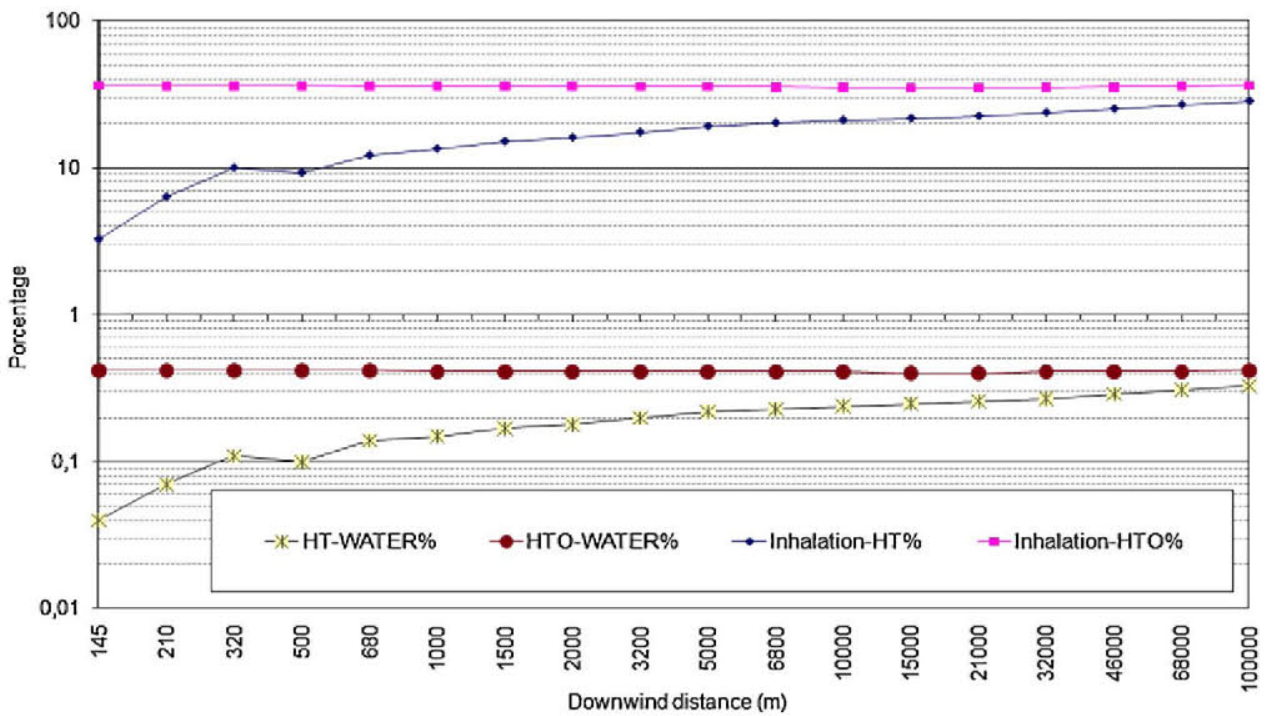

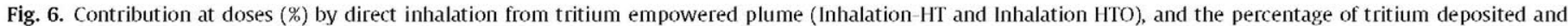
penet rated in subsoil (HT-water and HTO-water), into the $1-5 \mathrm{~cm}$ of depth soil, from $145 \mathrm{~m}$ to $100 \mathrm{~km}$ downwind distance. 


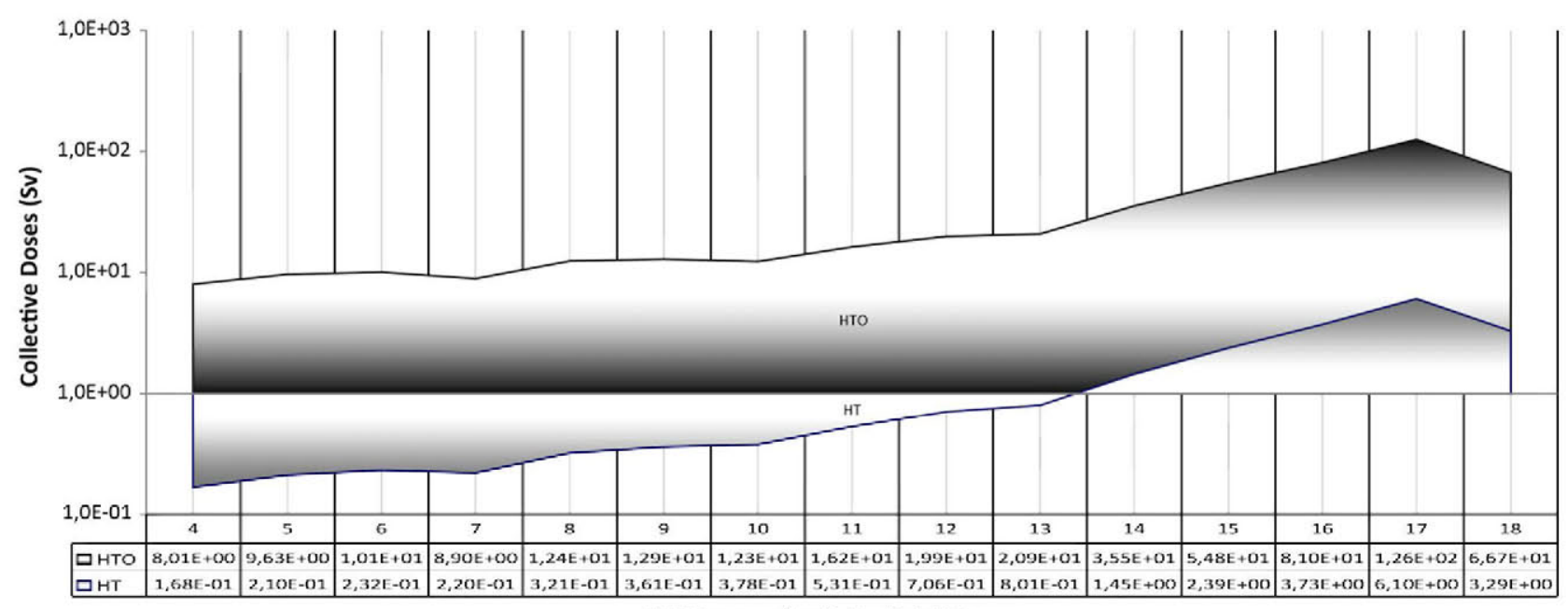

100 Persons/km2 - Radial distance

Fig. 7. Collective Effective Dose Equivalent (man-Sv) by radial distance and by sector of population.

are directly obtained from the plume. In all of the cases they are calculated for different populations, at several distances from source. In Fig. 5 NORMTRI makes evaluation of the major contribution to total doses coming from HT-Vegetation at distances from $145 \mathrm{~m}$ from reactor to $100 \mathrm{~km}$.

The maximum is close to the source reactor (contribution as much as $72 \%$ by Vegetation). There is a lineal decreasing of this contribution down to $60 \%$. HTO-Vegetation contribution remains constant as much as $50 \%$. Whereas HTO-milk is around $18 \%$ and just $8 \%$ is percentage for HTO-meat. Therefore the most important contribution at total doses coming from $\mathrm{HT}$ in vegetation.

\subsection{Contribution at doses (\%) by direct inhalation from tritium empowered plume}

NORMTRI makes forecast of the major contribution to total doses (\%) by direct inhalation from tritium empowered plume (Inhalation-HT and Inhalation HTO), and the percentage of tritium deposited and penetrated in subsoil (HT-water and HTO-water), into the $1-5 \mathrm{~cm}$ of depth soil, from $145 \mathrm{~m}$ to $100 \mathrm{~km}$ downwind distance (Fig. 6). As much as $40 \%$ comes from the inhalation of HTO. Initially close to reactor $(320 \mathrm{~m}) 10 \%$ comes from inhalation of HT. May be due to reemission of plume as much as $30 \%$ comes from inhalation of HT, at distances of $100 \mathrm{~km}$ from the source. The percentages of HTO-water and HT-water are very little in comparison with the inhalation, e.g. a constant percentage of $0,4 \%$ for HTOwater and variable inferior values for HT-water arriving to $0,3 \%$ in the case of HT-water.

\subsection{CEDE by sector around V-Plant}

In Fig. 7 Collective Effective Dose Equivalent (man-Sv) by radial distance and by sector of population is presented. Population may not suffer a significant radiological impact except for sector 17 , where collective dose is 126 man-Sv for HTO. Therefore there is a requirement to research individual doses before a possible decision on evacuation. May be it should be required in all or in part of sector 17 e.g. can affect to some persons on road traffic in A-7, AP-7 and 340 .

In sector 17 may be useful the real time HT concentration and dose rate ECMWF/Flexpart to achieve short term trends. Therefore in sector 17, may be useful the real time HT concentration and dose rate ECMWF/FLEXPART to achieve short term trends in dose rates.

\section{Conclusions}

HT and HTO are similar in NOMTRI and ECMWF/FLEXPART models except close reactor, where NORMTRI overestimates tritium concentration.

ECMWF/FLEXPART HT/HTO determines in accurate real time concentrations of atmospheric tritium. NORMTRI can be used from $10 \mathrm{~km}$ for accurate concentrations in surface land (already considering OBT).

For HT and HTO clouds over Mediterranean Basin there is a lineal decreasing evolution (in all levels).

In routine conditions of ITER the Most Exposed Individual (MEI) remains under the defined values into the Spanish protocol PENTA as precautionary measurements. Therefore, in level 3, EDEs remain under the threshold values for a possible confinement in the exclusion zone (zone 0). Further refinement may be based on Primary Phase outputs of ECMWF/FLEXPART. Maximum contribution at doses in vegetation (as much as $72 \%$ ) is close to reactor under $100 \mathrm{~km}$. Population may not suffer a significant radiological impact because NORMTRI output CEDES remains under the threshold values for a possible confinement in the exclusion zone (just considering skin transfer).

\section{References}

[1] P. Castro, M Velarde, J Ardao, J.M. Perlado, I Sedano, Validation of real time dispersion of tritium in ITER reactor over the Western Mediterranean Basin in different assessments, in: ICENES 2011 Proceedings, San Francisco(USA), May, 2011.

[2] P. Castro, M. Velarde, J. Ardao, J.M. Perlado, L. Sedano, Differences into HT and HTO concentrations in air into the Western Mediterranean Basin and Continental Europe and safety related issues, in: SOFE 2011, Chicago 26-June 2011, 2011.

[3] P. Castro, Analysis and weather prediction, in: Conference at Association Euratom CIEMAT - Laboratorio Nacional de Fusión, 2007.

[4] A. Persson, User Guide to ECMWF Forecast Products ECMWF, 2011

[5] A. Untch, Adiabatic formulation of the ECMWF model, ECMWF, Shinkfield Park, Reading RG2 9AX, UK, Room-11, (2011).

[6] Plan of Nuclear Emergency Exterior to Ascó and Vandellós (Tarragona), BOE no. 171,21 July 2006 , Sec. 1.

[7] A. Stohl, Technical Note: The Lagrangian Particle Dispersion Model FLEXPART Version 6.2, Norwegian Institute of Air Research, Kjeller, Norway, Organization, Vienna, Austria, Manuscript version from 21 April 2005.

[8] P. Castro, M. Velarde, J. Ardao, I.M. Perlado, L. Sedano, Consequences of different meteorological scenarios in the environmental impact assessment of tritium release 2P 01-09, Book of Abst racts 9th International Conference on Tritium Sci ence and Technology, TRITIUM 2010, October 24-29, 2010, Nara, Japan, Fusion Science and Technology, vol. 60, 2011, pp. 1284-1287.

[9] D. Arnold, A. Vargas, M. Montero, A. Dvorzhak, P. Seibert, Comparison of the dispersion model in RODOS-LX1 and MM5-V3.7-FLEXPART(V6.2). A case study 
for the nuclear power plant of Almaraz (Spain), Croatian Meteorological Journal 43 (2008) 485-490, ISSN: 1330-0083 (HARMO 12).

[10] P. Castro, M. Velarde, J. Ardao, J.M. Perlado, L. Sedano, Consequences of different meteorological scenarios in the environmental impact assessment of tritium release 2P 01-09, Book of Abstracts 9th International Conference on Tritium Science and Technology, TRITIUM 2010, October 24-29, 2010, Nara, Japan, FSD, 2010
[11] M. Velarde, J.M. Perlado, Tritium Dose Assessments under ITER Accidental/Normal Operational Conditions for the European Candidate Site of Vandellós (Spain), April 2003, Contract EISS-Vandellós.

[12] W. Raskob, NORMTRI a Computer Program for Assessing the Off_Site Consequences from Air-Borne Releases of Tritium during Normal Operation of Nuclear Facilities, Report FzK-5364, (1994) 